\title{
Characteristics and prediction of extreme drought event using LSTM model in Wei River Basin
}

\author{
Dongfei Yan $^{1}$, Rengui Jiang ${ }^{1,2, *}$, Jiancang Xie ${ }^{1}$, Yong Zhao ${ }^{2}$, Jiwei Zhu ${ }^{1}$, and Jichao Liang ${ }^{1}$ \\ ${ }^{1}$ State Key Laboratory of Eco-hydraulics in Northwest Arid Region of China, Xi' an University of Technology, Xi'an, China \\ ${ }^{2}$ State Key Laboratory of Simulation and Regulation of Water Cycle in River Basin, China Institute of Water Resources and \\ Hydropower Research, Beijing, China
}

\section{Article history: \\ Received 21 October 2020 \\ Revised 8 January 2021 \\ Accepted 7 February 2021}

Keywords:

Extreme drought event, SPEI, Nonparametric Mann-Kendall analysis, LSTM, Wei River Basin

\section{Citation:}

Yan, D., R. Jiang, J. Xie, Y. Zhao, J. Zhu, and J. Liang, 2021: Characteristics and prediction of extreme drought event using LSTM model in Wei River Basin. Terr. Atmos. Ocean. Sci., 32, 261-274, doi: 10.3319/TAO.2021.02.07.01

\begin{abstract}
As one of the natural disasters, drought has caused a large amount of financial loss in the past few centuries. It is quite essential to reveal the variation of extreme drought event in Wei River Basin (WRB) of China. This paper investigated the change patterns of extreme drought event using Standardized Precipitation Evapotranspiration Index (SPEI). Furthermore, the SPEI is predicted by combining different influencing factors in the WRB using Long Short-Term Memory (LSTM) model. The spatiotemporal variation characteristics were examined using non-parametric Mann-Kendall test, and the nonlinear relationships between El Niño-Southern Oscillation (ENSO) and SPEI were quantified using wavelet coherence analysis (WTC). Results showed that midland of the WRB have the highest probability of extreme drought events. Meanwhile, changes in SPEI in the northeast were more erratic than in other regions. The area with extreme drought had increased at a rate of $2.4 \%$ per decade. The prediction result of SPEI-24 was the best by LSTM model, and the prediction result of SPEI-3 was the worst. The R-square between the predicted value and the actual value of SPEI-24 is 0.87 . The results help to realize the characteristics of extreme drought in the last hundreds of years, which can provide scientific basis and reference for drought emergency response and management in the WRB.
\end{abstract}

\section{INTRODUCTION}

Drought refers to the climate phenomenon that water resources are no longer enough for human life and social development (Jiang et al. 2015; Zhao et al. 2019). Since ancient times, it has been one of the main natural disasters affecting the human beings, which exerted significant impacts on agriculture, ecology, environment, and economy (Dubrovsky et al. 2009). The main reason for severe damage and loss is that human beings have not fully grasped the occurrence rule and change trend of drought. With the continuous climate changes, many studies showed that the intensity and frequency of droughts had significantly increased across the world (Spinoni et al. 2015). Therefore, it is of great significance to study the regularity and change trend of drought occurrence for disaster prevention and mitigation. However, given the complex nature of drought, it is difficult to define drought events (Tirivarombo et al. 2018).

\footnotetext{
* Corresponding author

E-mail:jrengui@163.com
}

There are many studies using drought indices to simplify the description of complex drought phenomenon, and each drought index has its merits and demerits. As a common indicator, the Palmer Drought Severity Index (PDSI) has been widely used in meteorology, climate, agriculture, hydrology and water resources (Palmer 1968); The Standardized Precipitation Index (SPI) is an indicator of longterm climatic conditions relative to local rainfall, reflecting the occurrence probability of observed rainfall (McKee et al. 1993). Both drought indicators have been widely used, but there are still some deficiencies and limitations. The calculation of PDSI involves the lack of soil moisture, which leads to some uncertainties. The calculation of SPI is simple and convenient, but temperature and evaporation are not included. SPEI has the advantages of both PDSI and SPI (Vicente-Serrano et al. 2010). At present, SPEI has gradually become one of commonly used drought indicators to study the global drought change (Tan et al. 2015; Labudová 
et al. 2017; Abbasi et al. 2019). E.g., Wang et al. (2020) founded that the application of SPEI is superior to sc-PDSI and SPI in the northern Shaanxi province of China. Jiang et al. (2015) analyzed the variation features of drought using the SPI, SPEI, self-calibrated PDSI (scPDSI), and the original PDSI (orPDSI) in Shaanxi province, and founded that the SPEI is superior to scPDSI, SPI, and orPDSI.

At present, drought is influenced by the change of precipitation, evaporation, sunlight and other factors, and the abnormal phenomena of atmospheric circulation often causes the abnormal climate change. Many studies have shown that the climate change and climate oscillation are closely related (De Oliveira-Júnior et al. 2018; Singh and Shukla 2020; Yang et al. 2020). Liu et al. (2018b) analyzed the variation trends of annual maximum and minimum temperatures, as well as the teleconncection with sunspots number, soil moisture, and other factors in the WRB. Jiang et al. (2019) analyzed the variation trends of extreme precipitation indices (EPIs) and teleconnection between ENSO and EPIs in the WRB. Yan et al. (2020) analyzed the variation trends of extreme temperature indices (ETIs) and teleconnection between ENSO and ETIs in the WRB. At the same time, more studies use physical models to predict drought, while fewer use data-driven models to predict drought. At present, the data-driven model represented by deep learning has been widely used in the prediction and simulation of time series (Langkvist et al. 2014; Ismail Fawaz et al. 2019). However, there had few studies focused on the extreme drought events in the Wei River Basin (WRB) of China. By analyzing the response of SPEI to ENSO events, this paper aims to reveal the impact of ENSO on the drought changes, and try to use a data-driven model to predict and verify the drought index, so as to deeply understand the mechanism of extreme drought variation, which can provide decisionmaking support for the sustainable development and have important scientific value and practical significance.

\section{METHODOLOGY}

\subsection{Study Area}

The WRB flows through central Gansu, south-eastern Gansu and central Shaanxi provinces, and the area of WRB is about $135000 \mathrm{~km}^{2}$. Nearly half of the WRB are located in Shaanxi Province. The annual precipitation is about 500 - $800 \mathrm{~mm}$, most of them are short-term rainstorms, and frequent spring and summer droughts. The Jing River is the largest tributary of the Wei River, and the Beiluo River is the longest river in Shannxi province. JRB (the Jing River Basin) and BRB (the Beiluo River Basin) are important parts of the WRB. However, many droughts events happened in the WRB in recent years, the further study of temporal and spatial variation and frequency analysis of drought has a deeper meaning to the development of scientific drought prevention strategy (Huang et al. 2014; Jiang et al. 2020; Yan et al. 2020). The geographic of the WRB is shown in Fig. 1.

\subsection{Data Sources}

In this paper, the SPEI data for the period of 1901 2015 that is used to analyze the drought event can be downloaded at http://spei.csic.es/. It provides data on long periods

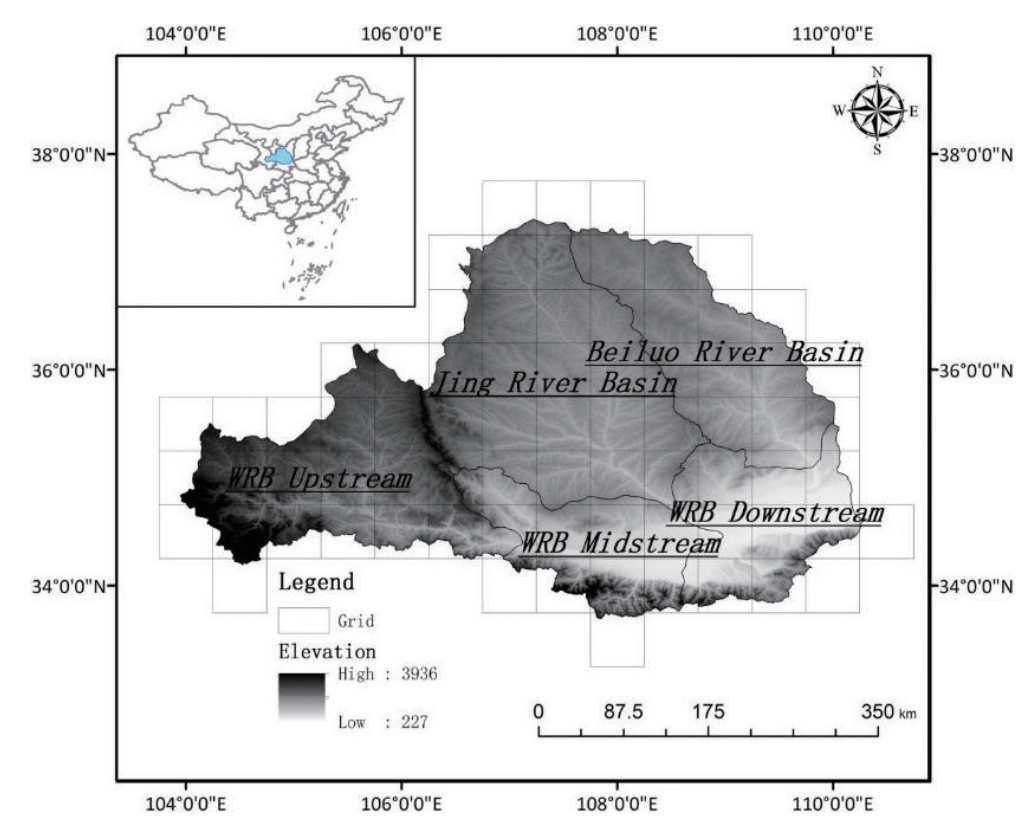

Fig. 1. The geographical information of the Wei River Basin. It shows that the Wei River Basin with girds that represent the locations of gridded data used in this study, with a total 75 grids at $0.5^{\circ} \times 0.5^{\circ}$ resolution. 
of drought around the world, the SPEI data sequence starts at $1^{\text {st }}$ January 1901 and ends at $31^{\text {st }}$ December 2015. The ENSO is measured using Niño 3.4 and SOI. The Niño 3.4 can be obtained from https://www.esrl.noaa.gov/. The SOI reflects the evolution of pressure enhancement and weakening on both sides of the Pacific, which can be obtained from https://crudata.uea.ac.uk/cru/data/soi/. Monthly temperature and precipitation data were downloaded at http://data.

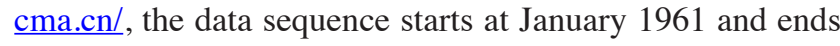
at December 2015.

\subsection{Standardized Precipitation Evapotranspiration Index (SPEI)}

SPEI was proposed mainly because that SPI does not consider evaportranspiration and PDSI does not have the multiple time scales. Monthly climatic water balance is calculated by Eq. (1).

$D_{i}=P_{i}-P E T_{i}$

where $P_{i}$ and $P E T_{i}$ are precipitation, potential evapotranspiration in period $i$. More details of SPEI can be found at previous studies (Vicente-Serrano et al. 2010; Jiang et al. 2015). The classification of drought and wetting according to the value of SPEI refer to previous of Yang et al. (2020).

\subsection{Mann-Kendall Trend Test}

The Mann-Kendall trend test has been widely used to detect the trend of time series (Du et al. 2015). This method is a non-parametric statistical test, which does not require samples to conform to some distributions and is not disturbed by a few outliers.

For a time series $A=\left\{a_{1}, a_{2}, \ldots, a_{m}\right\}$, the statistic value is given as follows:

$S=\sum_{i=1}^{m-1} \sum_{j=i+1}^{m} s\left(a_{j}-a_{i}\right)$

Where

$s\left(a_{j}-a_{i}\right)=\left\{\begin{array}{r}1, a_{j}-a_{i}>0 \\ 0, a_{j}-a_{i}=0 \\ -1, a_{j}-a_{i}<0\end{array}\right.$

The variance Var and statistic $Z$ are calculated as follows:

Var $=\frac{m(m-1)(2 m+5)}{18}$
$Z=\left\{\begin{array}{r}(S-1) / \sqrt{\operatorname{Var}}, S>0 \\ 0, S=0 \\ (S-1) / \sqrt{V a r}, S<0\end{array}\right.$

When $Z>0$, the trend of the sequence is upward, and vice versa (Yilmaz 2019). When $Z$ is greater than 1.65, 1.96, and 2.58 , it means that the significance test with confidence of 90,95 , and $99 \%$ has been passed respectively.

\subsection{Wavelet Coherence Analysis (WTC)}

Compared with the traditional wavelet transform, WTC can be used to analyse the cross-correlation between time series in time frequency. It has been widely used in many disciplines to find the common variation region of two-time series in the time-frequency space. For two data sequences $A$ and $B$, their wavelet transform were $W_{n}^{A}(S)$ and $W_{n}^{B}(S)$, the wavelet coherence defined by Eqs. (6) - (8):

$S(W)=S_{\text {scale }}\left\{S_{\text {time }}[W(s, t)]\right\}$

$\left.S(W)\right|_{s}=\left.\left[W(t, s) c_{1} e^{-\left(t^{2} / 2 s^{2}\right)}\right]\right|_{s}$

$\left.S(W)\right|_{n}=\left.\left[W(t, s) c_{2} \Pi(0.6 s)\right]\right|_{n}$

where $R_{n}^{2}(s) \in[0,1]$, and $W_{n}^{A B}(s)=W_{n}^{A}(s) \times W_{n}^{B}(s)$.

The WTC is calculated as follows:

$\phi_{n}(s)=\tan ^{-1}\left(\mathfrak{I}\left\{\left\langle s^{-1} W_{n}^{A B}(s)\right\rangle\right\} / \Re\left\{\left\langle s^{-1} W_{n}^{A B}(s)\right\rangle\right\}\right)$

where $\mathfrak{I}\{\ldots\}$ is the imaginary part of the wavelet spectra, and $\Re\{\ldots\}$ is the real part of wavelet spectra (Torrence and Compo 1998; Grinsted et al. 2004).

\subsection{Long Short-Term Memory (LSTM) Model}

An ensemble surrogate can improve the robustness and accuracy of the simulation model (Xing et al. 2019). The LSTM has been used in different aspects including learning translation language, controlling robots, image recognition, speech recognition, predicting time series data, and synthesizing music. The LSTM settles the long-term dependency problems of the recurrent neural network (RNN), Bengio et al. (1994) found that the long-term dependency is what makes RNN difficult to train. Figure 2 shows the differences in each cell between RNN and LSTM. The structure of RNN looks relatively simple and the LSTM is controlled by three gates. The forget gate outputs a vector between 0 and 1 with information about $h_{t-1}$ and $x_{t}$, which indicating how 
much of the previous information is retained or discarded. It is calculated by Eq. (10):

$f_{t}=\sigma\left(W_{f} \cdot\left[h_{t-1}, x_{t}\right]+b_{f}\right)$

The input gate gets the information for $h_{t-1}$ and $x_{t}$ and then decides which information to update. It is calculated by the following formula:

$i_{t}=\sigma\left(W_{i} \cdot\left[h_{t-1}, x_{t}\right]+b_{i}\right)$

$\widetilde{C}_{t}=\tanh \left(W_{c} \cdot\left[h_{t-1}, x_{t}\right]+b_{c}\right)$

The output gate is used to control whether this content is treated as the current output. It is calculated by the following formula:

$C_{t}=f_{t} * C_{t-1}+i_{t} * \widetilde{C}_{t}$

\subsection{Model Design}

This paper develops a model for predicting the monthly SPEI value, this model uses $\mathrm{SPEI}_{\mathrm{T}-3}$ (the SPEI value at period T-3), $\mathrm{SPEI}_{\mathrm{T}-2}$ (the SPEI value at period T-2), $\mathrm{SPEI}_{\mathrm{T}-1}$ (the SPEI value at period T-1), $\mathrm{PRE}_{\mathrm{T}}$ (the precipitation at period $\mathrm{T}$ ), and $\mathrm{TEM}_{\mathrm{T}}$ (the temperature at period $\mathrm{T}$ ) to predict $\mathrm{SPEI}_{\mathrm{T}}$ (the SPEI value at period T). The two layers LSTM structure was adopted and a Dropout layer was added between the LSTM layers. The units of the first LSTM layer are designed to be 128 and the units of the second LSTM layer to be 64, the parameter for Dropout layer is set to 0.2 , this means that $20 \%$ of the information will be discarded during training to prevent overfitting. The dense layer used the ReLU activation function, there has many papers have suggested that ReLU preforms better than either sigmoid or tanh function (Walls et al. 2020). Adam was chosen as the optimizer, which using momentum and adaptive learning rate to speed up convergence. Mean squared error (MSE) was selected as the losses of model. The number of iterations is determined by actual testing to ensure that the model is sufficiently learned.

\subsection{Research Framework}

In this study, statistical analysis and Mann-Kendall analysis were used to analyze the spatiotemporal variation characteristics of SPEI in the WRB, then WTC was used to analyze the potential teleconnection relationship between SPEI and ENSO, and finally LSTM model was used to predict the SPEI. The research framework of this paper is shown in Fig. 3.

\section{RESULTS}

\subsection{Spatiotemporal Variation Characteristics of SPEI in the WRB}

Statistical calculation is carried out on the time scale to obtain the proportions of each grid in different drought degrees in each year. The result shows that the proportions of drought in different years of the WRB vary in different time scales. Taking SPEI-12 for an example, there were few droughts events in the WRB from 1901 to 1916. However, there were more droughts in the following years, especially in 1927, 1948, and 1985, the proportion of droughts in the WRB is relatively large in these three years, and more than $70 \%$ of the grid areas have droughts of different degrees. Severe droughts occurred during 1920 to 1943,1952 to 1982, and 1985 to 2015. However, moderate drought and mild drought have maintained a certain proportion during 1920 to 2015 , especially after 2000 .

The spatial distributions of drought frequency were further analyzed. Figure 4 shows the drought occurrence probability in the WRB. Taking SPEI-12 for an example, the frequency of different drought conditions in all grids in the WRB was counted, the probability of drought occurrence in each grid was calculated, and the spatial drought distributions in the WRB were obtained by using the natural neighborhood method for spatial interpolation.

The northern part of the WRB is the place with the lowest occurrence probability of extreme drought and extreme wetting. The central part of the WRB is the Guanzhong plain area with the highest occurrence probability of extreme drought. Meanwhile, the western and eastern parts of the upper reaches of the WRB have a high occurrence probability of extreme drought, while the north-east parts of the upper reaches and the western parts of the lower reaches have a high occurrence probability of extreme wetting. The central part of the upper reaches and the western part of the WRB downstream are the areas with a high probability of severe drought, while the probability of severe drought is relatively low near the Guanzhong plain, at the same time, the WRB has a high probability of severe wetting except the northeast edge, and the other marginal parts (the west of the upper reaches, the south of the middle reaches, the east of the lower reaches, the north of the JRB and the north of the BRB) have a low probability of severe wetting. In the southern part of the WRB, the probability of moderate drought is low, while in the northern part of BRB, the probability of moderate drought is high. The middle part of the WRB has the highest probability of mild drought, while the western part has the lowest probability. The central WRB has the highest probability of mild drought, while the southern edge of the WRB has the lowest probability.

In order to understand the stability of drought spatial 

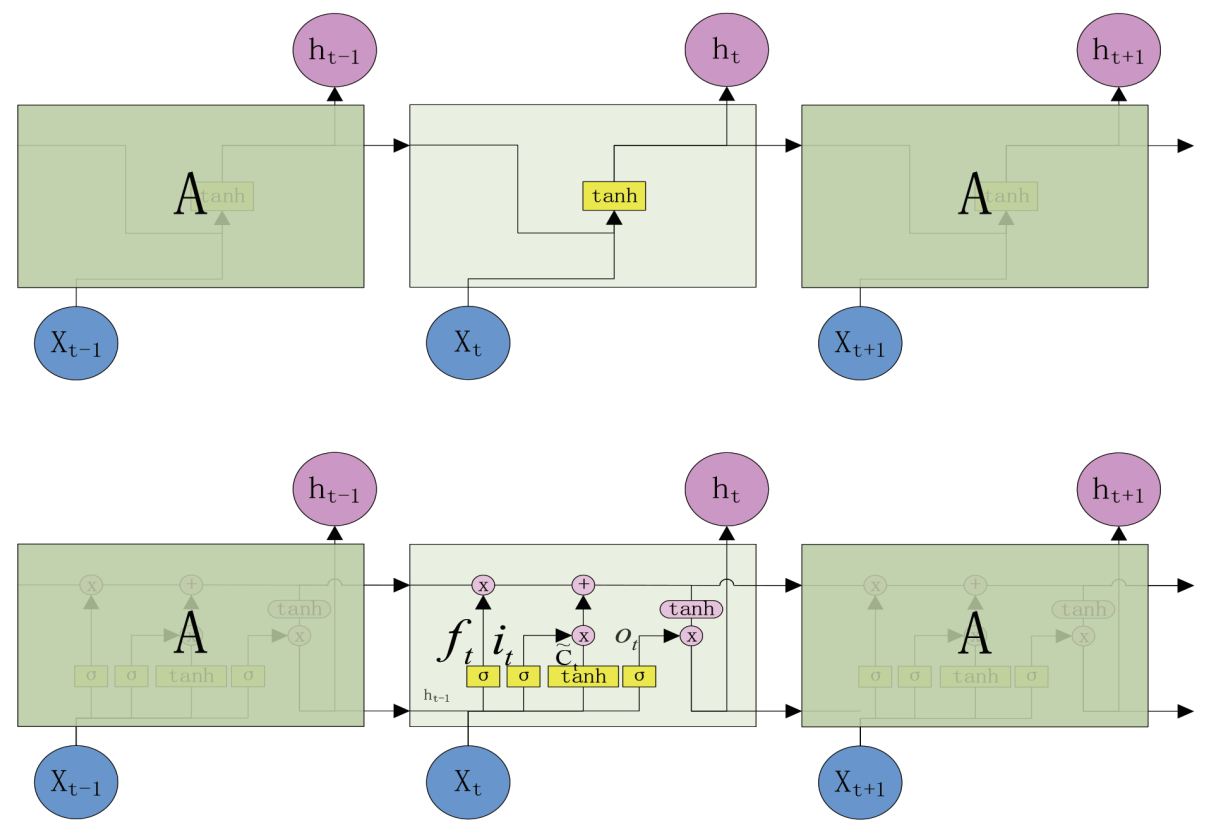

Fig. 2. Comparison of model structure between RNN and LSTM. RNN has only one tanh unit, while LSTM has multiple gates.

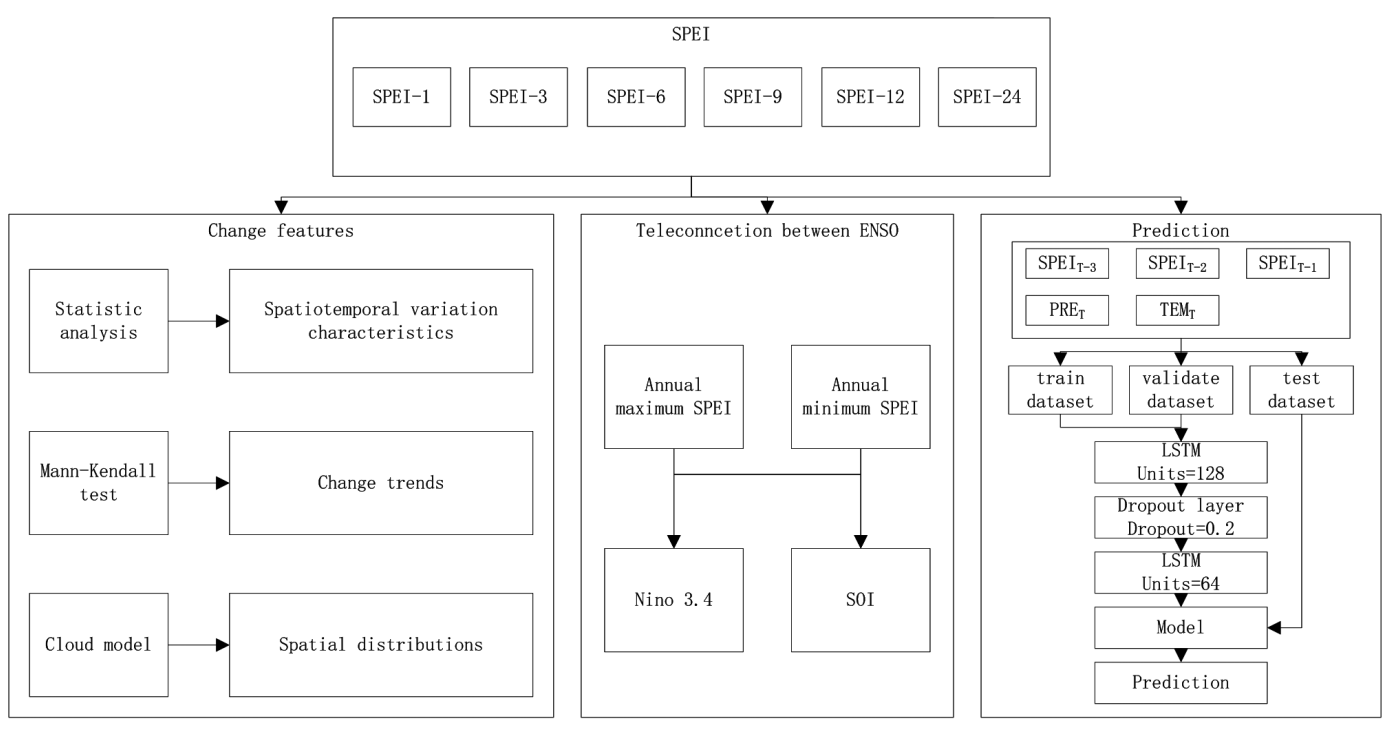

Fig. 3. The research route and framework of this study. 
(a)

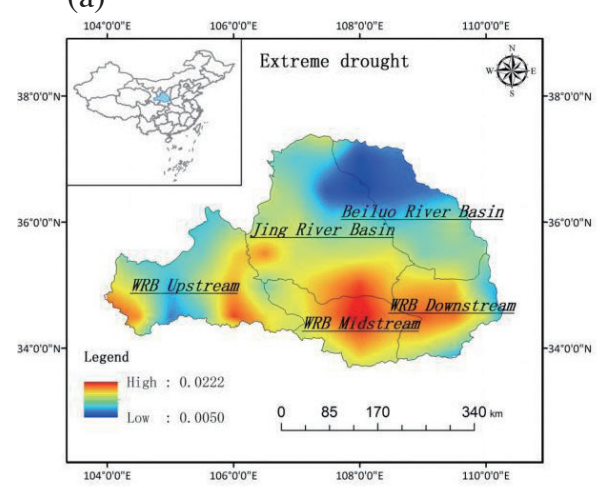

(c)



(e)

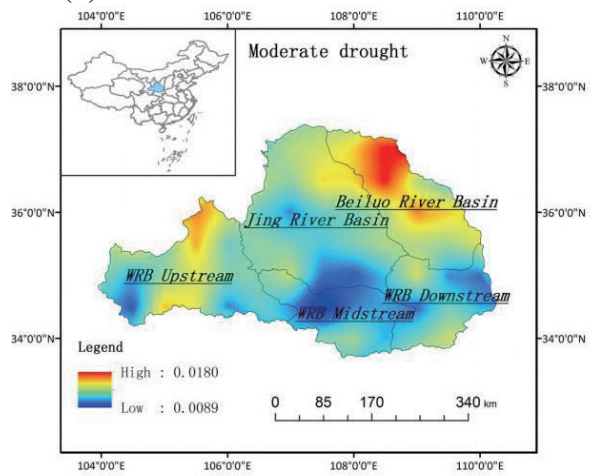

(g)

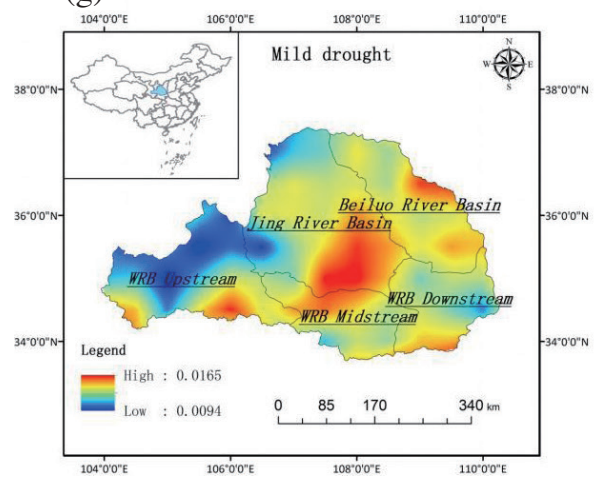

(b)

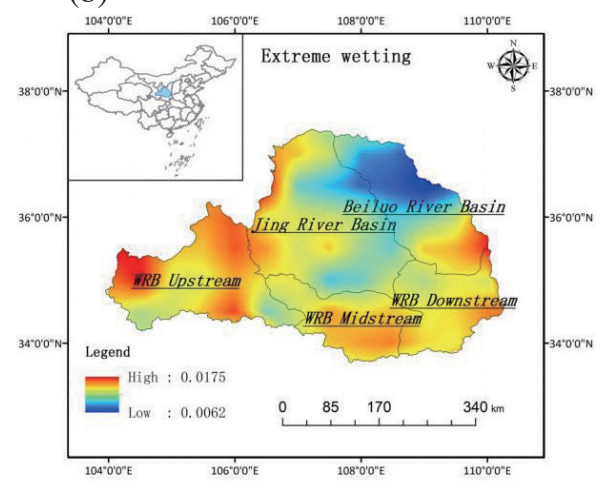

(d)

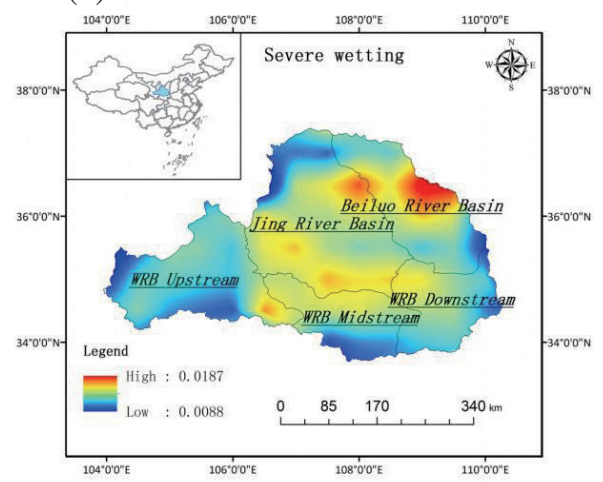

(f)

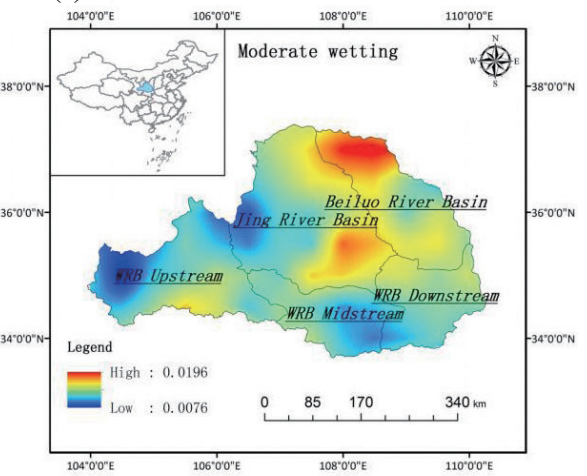

(h)

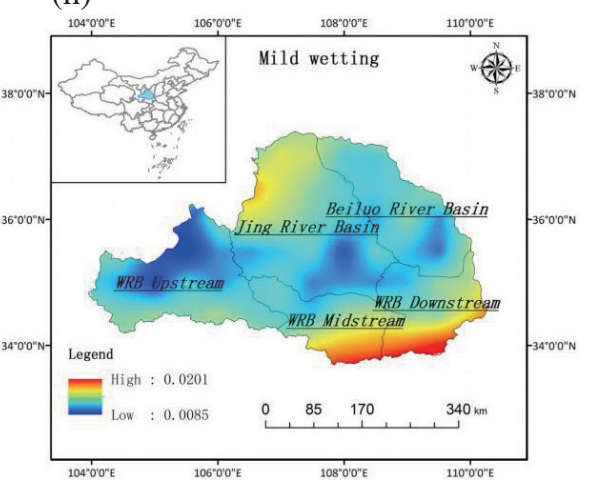

Fig. 4. The spatial distributions of different drought probability of SPEI-12 in the WRB: (a) Extreme drought; (b) Extreme wetting; (c) Severe drought; (d) Severe wetting; (e) Moderate drought; (f) Moderate wetting; (g) Mild drought; (h) Mild wetting. Under different drought conditions, the probability of drought in all grids was spatially interpolated. 
and temporal variation, the cloud model is used to analyze the drought variation of the WRB. Figure 5a shows that the Expectation (Ex) of SPEI-12 ranges from -0.0044 to 0.0025 in the WRB. The Ex of SPEI-12 in the western of WRB is significantly lower than other areas. Figure $5 \mathrm{~b}$ shows that the Entropy (En) in the northeast is higher than other regions, which indicates that the change in the northeast is more unstable. The result of the Hyper Entropy $(\mathrm{He})$ value is almost the opposite with respect to that of the En value, for example, the En value in the western of the WRB is the lowest in the whole region, but the He value is the highest in the whole region. The En value in Guanzhong plain is lower, but the He value is higher. There also have some areas where the exact opposite is not true. For example, the En value in the northeast of the WRB is the highest, but the He value is not the lowest. Instead, the peripheral areas around the region present the lowest He value. It indicates that the fluctuation of En in the upstream is higher than that in other areas.

\subsection{Trend Analysis of SPEI}

Figure 6 shows the spatial distribution of Mann-Kendall test results in the WRB. It shows that SPEI in different time scales have similar results in the period from 1901 to 2016. The Mann-Kendall test result of SPEI in the WRB showed a significant downward trend in Figs. 6a and b. Except the southeastern part of the WRB, the decreasing trend is significant at elsewhere. Meanwhile, the area in which SPEI-6 failed the significance test was larger than that in SPEI-1. The area that showed an upward trend was small in Fig. 6c, and it became larger in Fig. 6d. In Fig. 6c, the northern part of the WRB showed an upward trend. And the southeastern region showed significantly increasing trend in Fig. 6d. As the SPEI timescale increased, the trend of SPEI went from a significant decrease to a significant increase, first in the southeast of the WRB and then in the north of the WRB, indicating that SPEI values in these areas have increased and drought conditions have aggravated.

For further understanding the occurrence of extreme drought in the WRB, the extreme SPEI-12 values were statistically analyzed from different time scales and the trends analysis were carried out. The maximum SPEI value (MAX_ SPEI) and minimum SPEI value (MIN_SPEI) of all grids in each year were selected to form two sequences. Table 1 shows that the statistics show a significant increase in the maximum value $(\mathrm{P}<0.01)$ and a significant decrease in the minimum value $(\mathrm{P}<0.01)$, which indicates that trends are increasing, and there is a tendency to become more extreme.

\subsection{Teleconnection Between the Large-Scale Climate Factors and SPEI}

There are many reasons for changes in drought charac- teristics, such as human activity and climate impacts. From the perspective of atmospheric general circulation model, this paper further analyzed the potential relationships between the maximum and minimum SPEI and Niño 3.4, SOI using WTC analysis. In order to understand the potential correlation between atmospheric circulation factors and extreme drought, extreme SPEI values (maximum and minimum) including SPEI-3, and SPEI-12 were selected and wavelet coherence was used for analysis.

The WTC analysis between extreme SPEI and Niño 3.4 are shown in Fig. 7. Figure 7a shows that there is a $2-4$ years signal from 1959 to 1969 and a 0 - 2 years signal from 1995 to 2001 with negative correlations between Niño 3.4 and MAX_SPEI-3. Figure 7b shows that there is a 8 - 18 years signal from 1958 to 2010 with positive correlations between Niño 3.4 and MAX_SPEI-12. Figure 7c shows that there is a $0-2$ year signal during 1960 to 1965,1970 to 1974, and 1978 to 1985 and a 4 - 6 years signal from 1980 to 1987 with a $90^{\circ}$ phase difference between Niño 3.4 and MIN_SPEI-3. Figure 7d shows that there is a 5 - 7 year signal from 1976 to 1988 and a 7 - 10 year signal during 1955 to 1970 with negative correlations between Niño 3.4 and MIN_SPEI-12.

The WTC analysis results between SOI and extreme SPEI are shown in Fig. 8. Figure 8a shows that there is a 0 - 2 years signal from 1995 to 2000 with positive correlations and a 4 - 6 years signal from 1975 to 1985 with $-45^{\circ}$ phase difference between MAX_SPEI-3 and SOI. Figure $8 \mathrm{~b}$ shows that there is a Fig. 8c shows that there is a $2-3$ year signal from 1945 to 1950 with negative correlations and a 4 - 6 year signal from 1980 to 1990 with positive correlations between MIN_SPEI-3 and SOI. Figure 8d shows that there is a 5 - 6 year signal from 1980 to 1990 with positive correlations between MIN_SPEI-12 and SOI.

\subsection{Prediction of SPEI by Using LSTM}

Inputs to the model are $\mathrm{SPEI}_{\mathrm{T}-3}, \mathrm{SPEI}_{\mathrm{T}-2}, \mathrm{SPEI}_{\mathrm{T}-1}$, $\mathrm{PRE}_{\mathrm{T}}$, and $\mathrm{TEM}_{\mathrm{T}}$, the output is $\mathrm{SPEI}_{\mathrm{T}}$. The whole data set was divided to the ratio of $8: 1: 1$, the first $80 \%$ data and the middle $10 \%$ data were used as training sets verification sets, the last $10 \%$ data are used to predict and prevent models from overfitting.

Figure 9a shows that the model clearly captures the change trend of SPEI-1, but the prediction of the extreme values is not precise enough, the actual value greater than 2 (the Extremely wetting) occurred four times, but only one predicted value was also greater than 2 , and the other three extremely wet were all predicted to be the Severe wetting. Figure $9 \mathrm{~b}$ shows that compared to SPEI on other time scales, the model predicted SPEI-3 to be the worst, values and trends are less well predicted. Compared with the Figs. 9c, d, and e, it can be seen that the prediction of trend change and extreme value in Fig. 9f is more accurate. The 
evaluation indices (MSE and $\mathrm{R}^{2}$ ) can also reflect that SPEI24 has the best prediction performance as shown in Table 2 . Table 2 shows that the best model of prediction is SPEI-24, the MSE and $\mathrm{R}^{2}$ are $0.2342 \mathrm{~m}^{3} \mathrm{~s}^{-1}$ and 0.8709 , it is better than the other models. And the worst model is SPEI-3, the MSE and $\mathrm{R}^{2}$ are $0.5929 \mathrm{~m}^{3} \mathrm{~s}^{-1}$ and 0.3977 , it is worse than other models.

\section{DISCUSSION}

This paper first analyzes the spatial and temporal variation of drought in the WRB. It was found that the study area showed a general trend of drying out by Mann-Kendall test. Zou et al. (2017) founded that a trendency to dry out can be observed during 1961 to 2012 in the WRB, this is consistent with the findings of this paper. The results also found that both the accumulation time of extreme drought and the maximum time of extreme drought in Guanzhong Plain were much higher than those in other regions, which indicated that the drought situation in Guanzhong area was more severe. It was worthy of attention to ensure the water supply in this area, which was consistent with the previous findings of Huang et al. (2014).

This paper further explains the variation trend and potential influencing factors of extreme drought events in
WRB by analyzing the teleconnection between SPEI and ENSO. The results show that there are complex correlations between SPEI and atmospheric circulation patterns. The correlation between SPEI and Niño 3.4 and SOI had opposite results, this may be due to the negative SOI corresponds to an El Niño event, while the continuous increase of Niño 3.4 is also considered to be an El Niño event. At present, there are many atmospheric circulation patterns have been used to realize the impacts of climate change, such as North Atlantic Oscillation (NAO) (Vazifehkhah and Kahya 2018), Altantic Multidecadal Oscillation (AMO) (Schillerberg and Tian 2020), Arctic Oscillation (AO) (Li et al. 2017), and Pacific decadal Oscillation (PDO) (Oñate-Valdivieso et al. 2020). Given the close relationships between atmospheric circulation patterns, how to realize the impact of atmospheric anomalies on the change of extreme drought events needs more attention.

LSTM captures the changing trend of time series data accurately, but there are still some problems worth further studying and discussing. In this study the best predicted result is SPEI-24, and it can still be seen from the Fig. 9f that there are still three distinct mild and moderate droughts that are not accurately predicted. This problem of inaccurate predictions of extreme values is more pronounced in SPEI3 . There are many variation trend prediction errors and

(a)

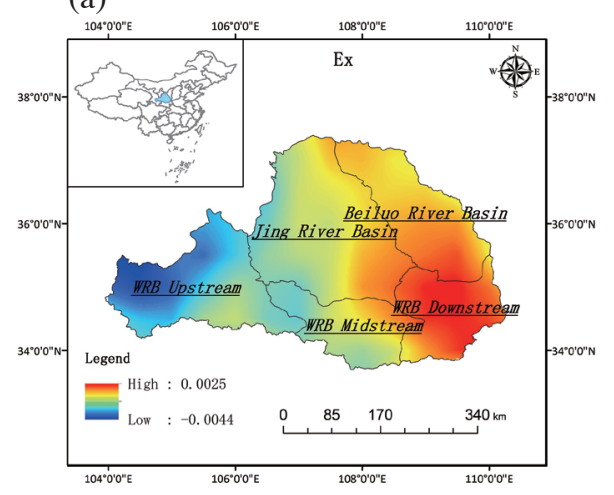

(b)

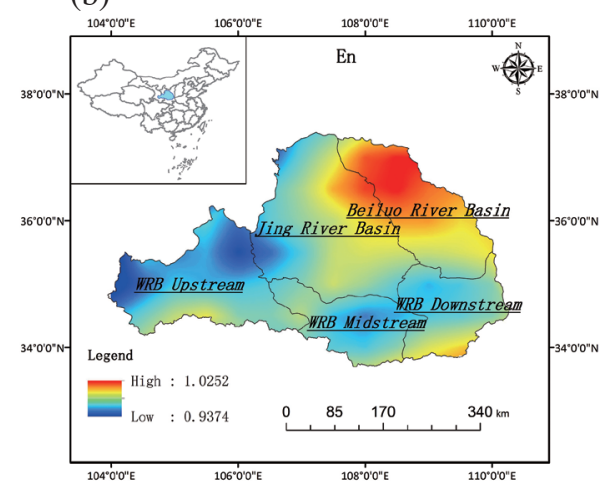

(c)

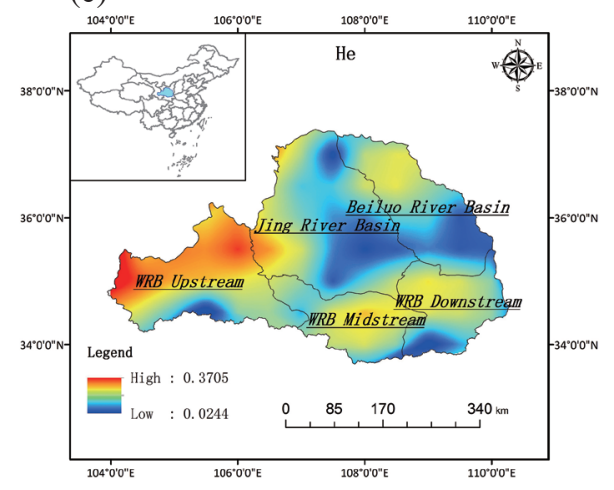

Fig. 5. The spatial variation distributions of three numeric characteristic (Ex, En, He) in the WRB: (a) Ex; (b) En; (c) He. Ex refers to the expectation and represents the average level of numerical change; En refers to entropy, which is used to indicate the stability of numerical changes; He refers to the super entropy and is used to indicate the stability of the change in En. 
(a)

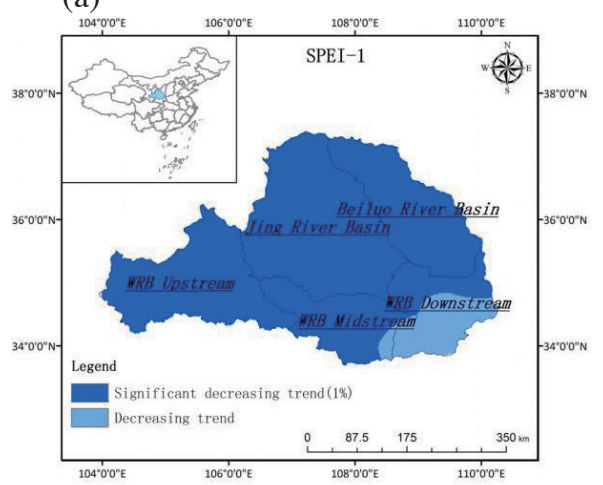

(c)

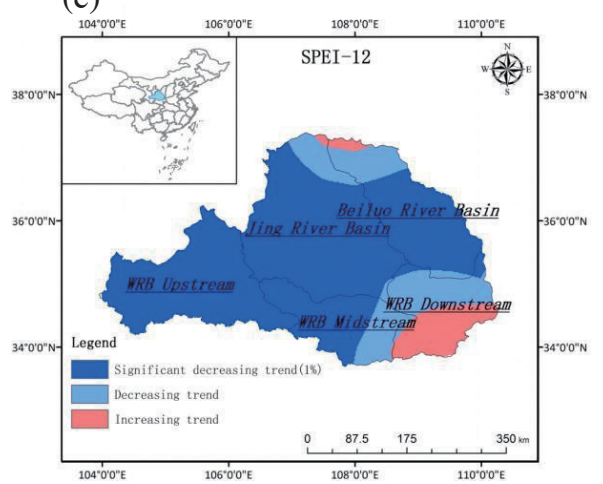

(b)

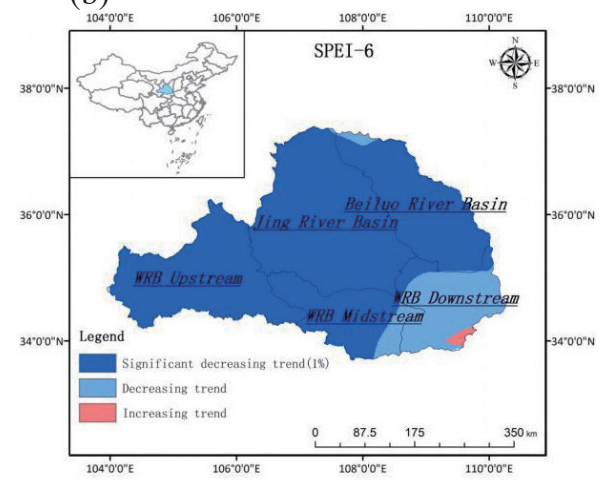

(d)

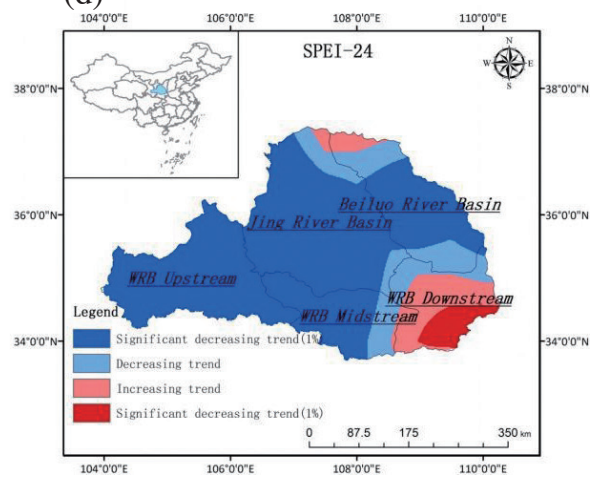

Fig. 6. The spatial distribution of Mann-Kendall test results in the WRB: (a) SPEI-1; (b) SPEI-6; (c) SPEI-12; (d) SPEI-24. The Mann-Kendall test statistics of all grids was interpolated spatially.

Table 1. Mann-Kendall test statistics of the maximum and minimum sequence of SPEI at different SPEI time scales. If the absolute value of the statistic is greater than 2.32 , it is statistically significant at the $1 \%$ significance level. A positive (negative) value represents a trend of increase (decrease).

\begin{tabular}{c|cc}
\hline & MAX_SPEI & MIN_SPEI \\
\hline SPEI-1 & $6.3522^{* *}$ & $-6.6399 * *$ \\
SPEI-3 & $5.9437 * *$ & $-5.777 * *$ \\
SPEI-6 & $5.9026^{* *}$ & $-5.4482^{* *}$ \\
SPEI-9 & $5.3346^{* *}$ & $-4.8488^{* *}$ \\
SPEI-12 & $5.3153^{* *}$ & $-5.1171^{* *}$ \\
SPEI-24 & $4.9369^{* *}$ & $-5.554 * *$ \\
\hline
\end{tabular}

Note: * and $* *$ represents statistically significant trend at significant level of 5\% and $1 \%$. 

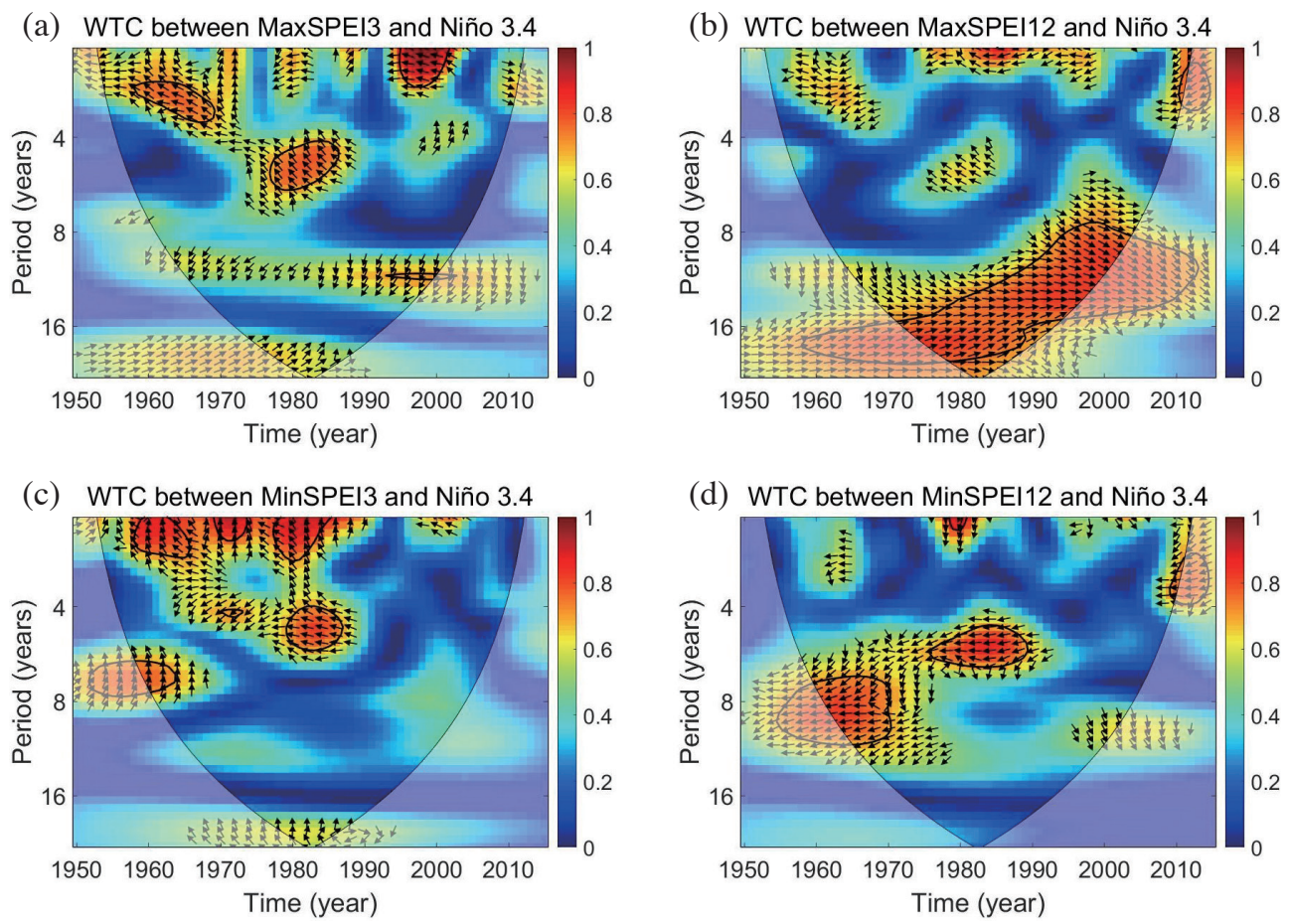

Fig. 7.WTC analysis results between Niño 3.4 and extreme value of SPEI: (a) MAX_SPEI-3,(b) MAX_SPEI-12,(c) MIN_SPEI-3,(d) MIN_SPEI12. The thick enclosed areas indicate statistically significance level of 5\% against a red noise process, and the arrows in the figures represent the phase difference of time periods and coherence bigger than 0.5 between two data series, with anti-phase pointing left and in phase pointing right
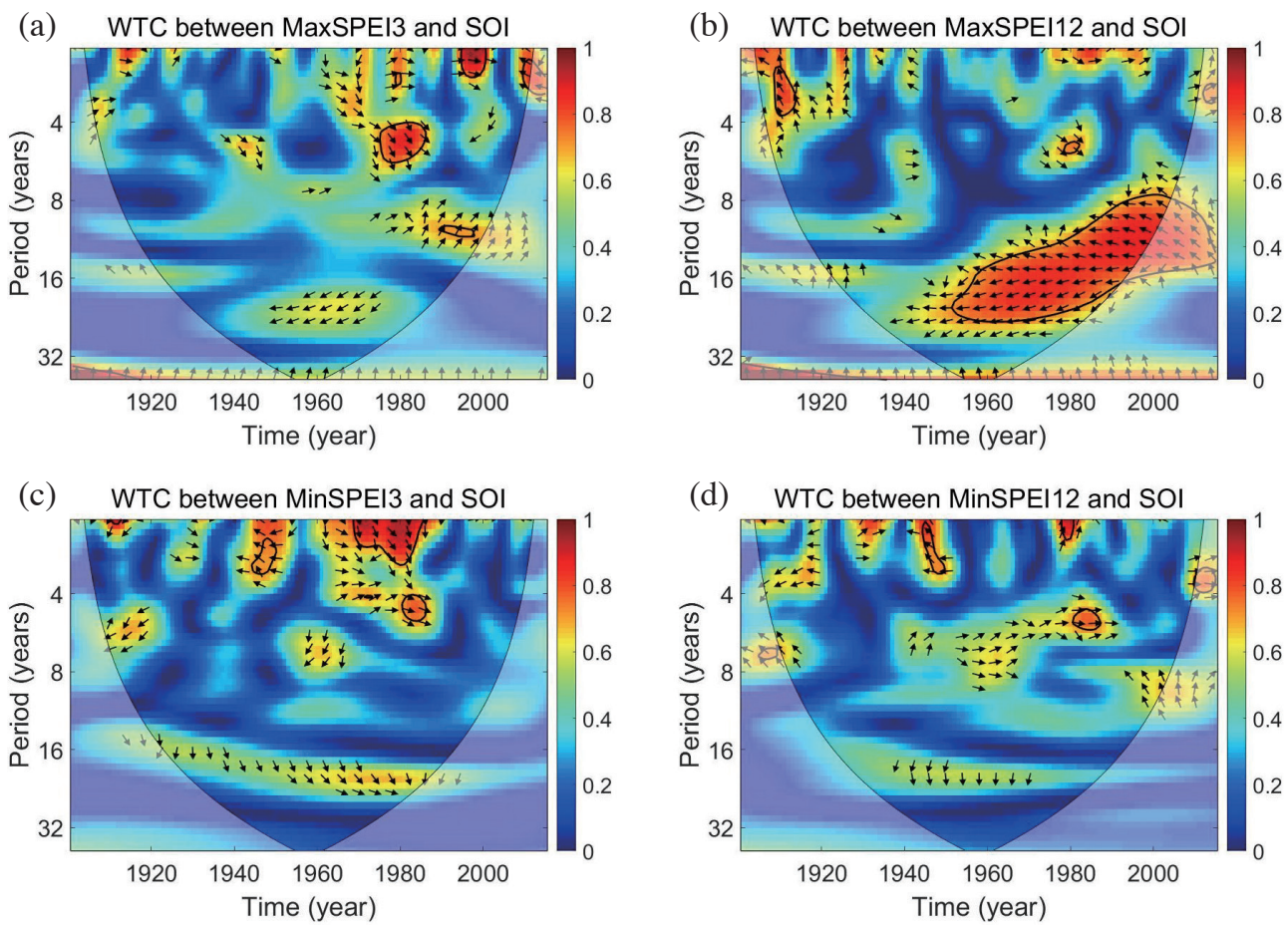

Fig. 8. WTC analysis results between SOI and extreme value of SPEI: (a) MAX_SPEI-3, (b) MAX_SPEI-12, (c) MIN_SPEI-3, (d) MIN_SPEI-12. 
(a)

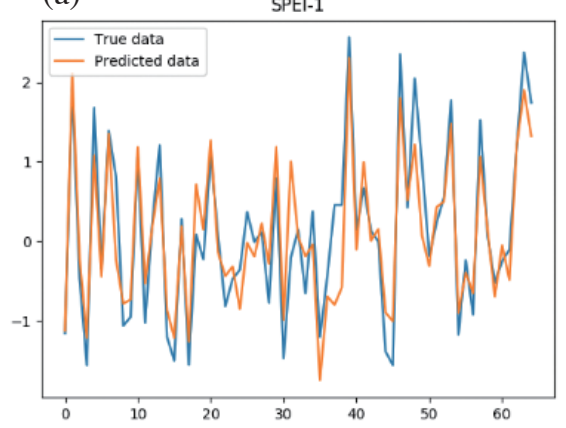

(c)

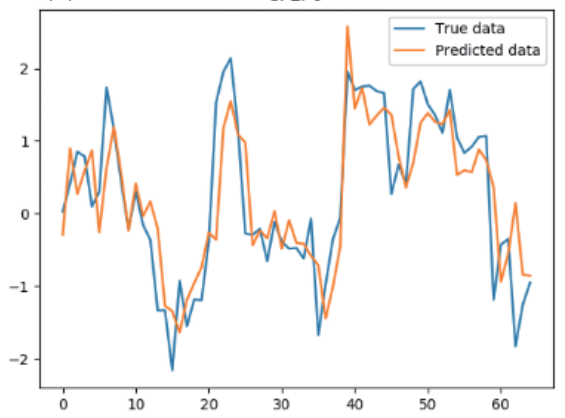

(e)

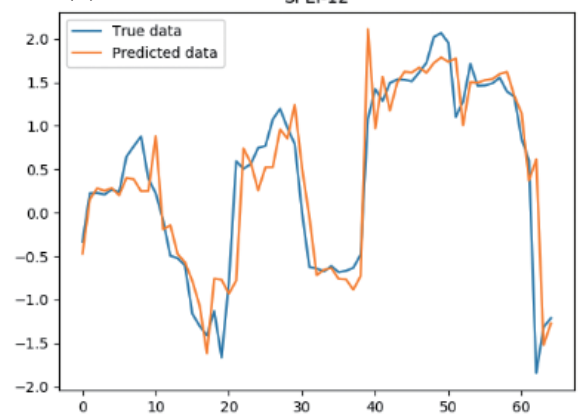

(b)

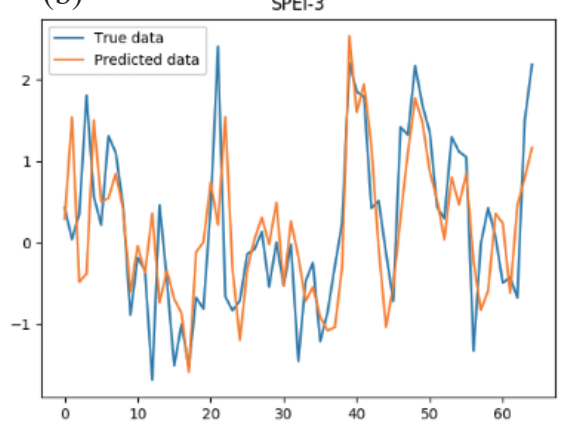

(d)

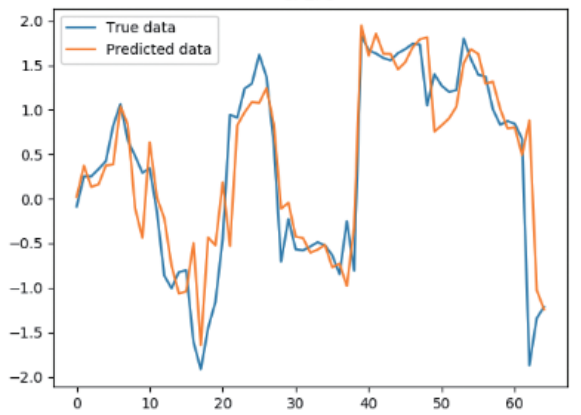

(f) SPEI-24

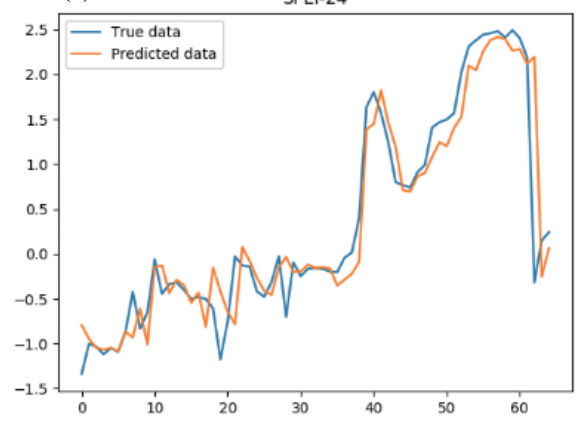

Fig. 9. The comparison between the predicted value and the true value: (a) SPEI-1; (b) SPEI-3; (c) SPEI-6; (d) SPEI-9; (e) SPEI-12; (f) SPEI-24.

Table 2. The evaluation results of different models. The smaller the MSE indicates the smaller prediction error. The closer $\mathrm{R}^{2}$ to 1 indicates the better model fits.

\begin{tabular}{c|cc}
\hline & MSE $\left(\mathbf{m}^{3} \mathbf{s}^{-1}\right)$ & $\mathbf{R}^{2}$ \\
\hline SPEI-1 & 0.3532 & 0.8236 \\
SPEI-3 & 0.5929 & 0.3977 \\
SPEI-6 & 0.4552 & 0.6865 \\
SPEI-9 & 0.3309 & 0.7519 \\
SPEI-12 & 0.2900 & 0.7926 \\
SPEI-24 & 0.2342 & 0.8709 \\
\hline
\end{tabular}


extreme value prediction errors in SPEI-3, and the reasons for these needs to be further studied and discussed. Similar to the LSTM, which is a variant of RNN, the LSTM has its own variants, the most famous of which is the gate recurrent unit (GRU) (Liu et al. 2018a), more variations can be designed around the theme of gating. Some scholars have found that adding a bias to the forgetting gate in the LSTM will make the model more robust (Gers et al. 2000).

However, although the complexity of LSTM model layers increases, the parameters also increase greatly. The design of the model and the tuning of parameters have a huge influence on the application of the model. In this paper, there is no special detail tuning for all parameters. In the future, further research may be conducted on parameter tuning to improve the robustness of the model.

\section{CONCLUSION}

In this paper, SPEI was selected as the indicator to evaluate extreme drought events during 1901 to 2016 in the WRB. Mann-Kendall trends test method was used to investigate the spatiotemporal variation characteristics of WRB extreme drought events, and WTC analysis was used to analyze the teleconnection between SPEI and ENSO. The main conclusions of this paper are as follows:

(1) On the temporal scale, extreme droughts and extreme wetting were almost nonexistent between 1901 and 1920. It can be roughly divided into three stages from 1920. During the first period of 1920 - 1940, extreme drought events are beginning to increase. During the second period of 1940 - 1980, drought events were rare in the early stage, and frequent in the latter. During the last period of 1980 - 2015, extreme drought events happened frequently before 2010 and rarely after 2010 . The total drought area had increased at a rate of $3.96 \%$ / decade, and the extreme drought area had increased at a rate of $2.4 \%$ per decade. On the spatial scale, the northern part of the WRB has the lowest probability of extreme drought and extreme wetting. The central part of the WRB, namely the Guanzhong plain, has the highest probability of extreme drought. As the most important grain producing area in Shaanxi province, the relevant departments should pay attention to the drought conditions of Guanzhong Plain.

(2) The MAX_SPEI and MIN_SPEI of the WRB had a significant increasing trend and a significant decreasing trend, respectively, indicating that extreme wetting and drought in the WRB may be polarized in the future. The teleconnection between SPEI and SOI is mainly positive, while the teleconnection between SPEI and Niño 3.4 is mainly negative.

(3) Through the prediction of LSTM model, it is found that the prediction result of SPEI-24 is the best, and SPEI3 is the worst. The MSE of SPEI-24 and SPEI-3 are
$0.2343 \mathrm{~m}^{3} \mathrm{~s}^{-1}$ and $0.5929 \mathrm{~m}^{3} \mathrm{~s}^{-1}$, and $\mathrm{R}^{2}$ of SPEI-24 and SPEI-3 are 0.8709 and 0.3977 .

(4) In particular, the teleconnection between ENSO and extreme drought events was analyzed, which provides ideas for the influence of atmospheric circulation anomalies on the occurrence of drought events.

Acknowledgements This paper was partly funded by the National Key Research and Development Program of China (Grant No. 2016YFC0401409), National Natural Science Foundation of China (Grant No. 51679188, 51509201), Young Technology Star in Shaanxi Province of China (Grant No. 2020KJXX-092). We sincerely appreciate the editor and anonymous reviewers.

\section{REFERENCES}

Abbasi, A., K. Khalili, J. Behmanesh, and A. Shirzad, 2019: Drought monitoring and prediction using SPEI index and gene expression programming model in the west of Urmia Lake. Theor. Appl. Climatol., 138, 553-567, doi: 10.1007/s00704-019-02825-9. [Link]

Bengio, Y., P. Simard, and P. Frasconi, 1994: Learning long-term dependencies with gradient descent is difficult. IEEE Trans. Neural Netw., 5, 157-166, doi: 10.1109/72.279181. [Link]

De Oliveira-Júnior, J. F., G. De Gois, P. M. de Bodas Terassi, C. A. da Silva Junior, C. J. C. Blanco, B. S. Sobral, and K. A. C. Gasparini, 2018: Drought severity based on the SPI index and its relation to the ENSO and PDO climatic variability modes in the regions North and Northwest of the State of Rio de Janeiro - Brazil. Atmos. Res., 212, 91-105, doi: 10.1016/j.atmosres.2018.04.022. [Link]

Du, S., H. Gu, J. Wen, K. Chen, and A. Van Rompaey, 2015: Detecting Flood Variations in Shanghai over 1949-2009 with Mann-Kendall Tests and a Newspaper-Based Database. Water, 7, 1808-1824, doi: 10.3390/w7051808. [Link]

Dubrovsky, M., M. D. Svoboda, M. Trnka, M. J. Hayes, D. A. Wilhite, Z. Zalud, and P. Hlavinka, 2009: Application of relative drought indices in assessing climatechange impacts on drought conditions in Czechia. Theor. Appl. Climatol., 96, 155-171, doi: 10.1007/ s00704-008-0020-x. [Link]

Gers, F. A., J. Schmidhuber, and F. Cummins, 2000: Learning to forget: Continual prediction with LSTM. Neural Comput., 12, 2451-2471, doi: 10.1162/089976600300015015. [Link]

Grinsted, A., J. C. Moore, and S. Jevrejeva, 2004: Application of the cross wavelet transform and wavelet coherence to geophysical time series. Nonlinear Process Geophys., 11, 561-566, doi: 10.5194/npg-11-5612004. [Link] 
Huang, S., J. Chang, Q. Huang, and Y. Chen, 2014: Spatiotemporal Changes and Frequency Analysis of Drought in the Wei River Basin, China. Water Resour. Manag., 28, 3095-3110, doi: 10.1007/s11269-014-0657-4. [Link]

Ismail Fawaz, H., G. Forestier, J. Weber, L. Idoumghar, and P.-A. Muller, 2019: Deep learning for time series classification: A review. Data Min. Knowl. Discov., 33, 917-963, doi: 10.1007/s10618-019-00619-1. [Link]

Jiang, R., J. Xie, H. He, J. Luo, and J. Zhu, 2015: Use of four drought indices for evaluating drought characteristics under climate change in Shaanxi, China: 1951-2012. Nat. Hazards, 75, 2885-2903, doi: 10.1007/s11069014-1468-x. [Link]

Jiang, R., Y. Wang, J. Xie, Y. Zhao, F. Li, and X. Wang, 2019: Assessment of extreme precipitation events and their teleconnections to El Niño Southern Oscillation, a case study in the Wei River Basin of China.Atmos. Res., 218, 372-384, doi: 10.1016/j.atmosres.2018.12.015. [Link]

Jiang, R., R. Cao, X. X. Lu, J. Xie, Y.Zhao, and F. Li, 2020: Quantifying precipitation extremes and their relationships with large-scale climate oscillations in a tropical country, Singapore: 1980-2018. Singap. J. Trop. Geogr., 41, 384-412, doi: 10.1111/sjtg.12329. [Link]

Labudová, L., M. Labuda, and J. Takáč, 2017: Comparison of SPI and SPEI applicability for drought impact assessment on crop production in the Danubian Lowland and the East Slovakian Lowland. Theor. Appl. Climatol., 128, 491-506, doi: 10.1007/s00704-016-1870-2. [Link]

Langkvist, M., L. Karlsson, and A. Loutfi, 2014: A review of unsupervised feature learning and deep learning for time-series modeling. Pattern Recognit. Lett., 42, 1124, doi: 10.1016/j.patrec.2014.01.008. [Link]

Li, J., Y. Lei, X. Liu, H. Mao, F. Chen, and B. A. Engel, 2017: Effects of AO and Pacific SSTA on severe droughts in Luanhe River basin, China. Nat. Hazards, 88, 1251-1267, doi: 10.1007/s11069-017-2917-0. [Link]

Liu, J., C. Wu, and J. Wang, 2018a: Gated recurrent units based neural network for time heterogeneous feedback recommendation. Inf. Sci., 423, 50-65, doi: 10.1016/j. ins.2017.09.048. [Link]

Liu, S., S. Huang, Y. Xie, Q. Huang, G. Leng, B. Hou, Y. Zhang, and X. Wei, 2018b: Spatial-temporal changes of maximum and minimum temperatures in the Wei River Basin, China: Changing patterns, causes and implications. Atmos. Res., 204, 1-11, doi: 10.1016/j. atmosres.2018.01.006. [Link]

McKee, T. B., N. J. Doesken, and J. Kleist, 1993: The relationship of drought frequency and duration to time scales. Proceedings of the 8th Conference on Applied Climatology, Anaheim, California, Vol. 17, 179-183.
Oñate-Valdivieso, F., V. Uchuari, and A. Oñate-Paladines, 2020: Large-Scale Climate Variability Patterns and Drought: A Case of Study in South - America. Water Resour. Manag., 34, 2061-2079, doi: 10.1007/s11269020-02549-w. [Link]

Palmer, W. C., 1968: Keeping track of crop moisture conditions, nationwide: The New Crop Moisture Index. Weatherwise, 21, 156-161, doi: 10.1080/00431672.1968.9932814. [Link]

Schillerberg, T. A. and D. Tian, 2020: Changes of crop failure risks in the United States associated with large-scale climate oscillations in the Atlantic and Pacific Oceans. Environ. Res. Lett., 15, 064035, doi: 10.1088/17489326/ab82cd. [Link]

Singh, R. M. and P. Shukla, 2020: Drought Characterization Using Drought Indices and El Niño Effects. Natl. Acad. Sci. Lett.-India, 43, 339-342, doi: 10.1007/ s40009-019-00870-6. [Link]

Spinoni, J., G. Naumann, J. Vogt, and P. Barbosa, 2015: European drought climatologies and trends based on a multi-indicator approach. Glob. Planet. Change, 127, 50-57, doi: 10.1016/j.gloplacha.2015.01.012. [Link]

Tan, C., J. Yang, and M. Li, 2015: Temporal-Spatial Variation of Drought Indicated by SPI and SPEI in Ningxia Hui Autonomous Region, China. Atmosphere, 6, 13991421, doi: 10.3390/atmos6101399. [Link]

Tirivarombo, S., D. Osupile, and P. Eliasson, 2018: Drought monitoring and analysis: Standardised Precipitation Evapotranspiration Index (SPEI) and Standardised Precipitation Index (SPI). Phys. Chem. Earth, 106, 1-10, doi: 10.1016/j.pce.2018.07.001. [Link]

Torrence, C. and G. P. Compo, 1998: A practical guide to wavelet analysis. Bull. Amer. Meteorol. Soc., 79, 6178, doi: 10.1175/1520-0477(1998)079<0061:APGTW A>2.0.CO;2. [Link]

Vazifehkhah, S. and E. Kahya, 2018: Hydrological drought associations with extreme phases of the North Atlantic and Arctic Oscillations over Turkey and northern Iran. Int. J. Climatol., 38, 4459-4475, doi: 10.1002/ joc.5680. [Link]

Vicente-Serrano, S. M., S. Beguería, and J. I. López-Moreno, 2010: A Multiscalar Drought Index Sensitive to Global Warming: The Standardized Precipitation Evapotranspiration Index. J. Clim., 23, 1696-1718, doi: 10.1175/2009JCLI2909.1. [Link]

Walls, S., A. D. Binns, J. Levison, and S. Macritchie, 2020: Prediction of actual evapotranspiration by artificial neural network models using data from a Bowen ratio energy balance station. Neural Comput. Appl., 32, 14001-14018, doi: 10.1007/s00521-020-04800-2. [Link]

Wang, X., L. Zhuo, C. Li, B. A. Engel, S. Sun, and Y. Wang, 2020: Temporal and spatial evolution trends of drought in northern Shaanxi of China: 1960-2100. Theor. Appl. 
Climatol., 139, 965-979, doi: 10.1007/s00704-01903024-2. [Link]

Xing, Z., R. Qu, Y. Zhao, Q. Fu, Y. Ji, and W. Lu, 2019: Identifying the release history of a groundwater contaminant source based on an ensemble surrogate model. J. Hydrol., 572, 501-516, doi: 10.1016/j.jhydrol.2019.03.020. [Link]

Yan, D. F., R. G. Jiang, J. C. Xie, Y. Zhao, J. C. Liang, and Y. P. Wang, 2020: Changes in Extreme Temperature Events and Their Relationships with the El Niño-Southern Oscillation in the Wei River Basin, China. Appl. Ecol. Environ. Res., 18, 2395-2412, doi: 10.15666/aeer/1802_23952412. [Link]

Yang, Y., T. Y. Gan, and X. Tan, 2020: Spatiotemporal changes of drought characteristics and their dynamic drivers in Canada. Atmos. Res., 232, 104695, doi: 10.1016/j.atmosres.2019.104695. [Link]

Yilmaz, B., 2019: Analysis of Hydrological Drought Trends in the Gap Region (Southeastern Turkey) by Mann-Kendall Test and Innovative Sen Method. Appl. Ecol. Environ. Res., 17, 3325-3342, doi: 10.15666/ aeer/1702_33253342. [Link]

Zhao, Q., C. H. Zou, K. F. Wang, Q. Gao, and T. Yao, 2019: Spatial and Temporal Distribution Characteristics of Drought and Its Influencing Factors in Heilongjiang Province, China from 1956 To 2015. Appl. Ecol. Environ. Res., 17, 2631-2650, doi: 10.15666/ aeer/1702_26312650. [Link]

Zou, L., J. Xia, and D. She, 2017: Drought Characteristic Analysis Based on an Improved PDSI in the Wei River Basin of China. Water, 9, 178, doi: 10.3390/ w9030178. [Link] 